\title{
A Mathematical Model of the Nascent Cyclone
}

\author{
Kausik Kumar Majumdar
}

\begin{abstract}
In this letter, we have proposed a mathematical modeling of a disturbance created by winds coming from different directions and colliding to give rise to a vortex under certain conditions. Under favorable conditions, this vortex may lead to the development of a cyclonic storm (cyclogenesis).
\end{abstract}

Index Terms-Fuzzy systems, meteorology, simulation, storms, terrestrial atmoshpere, tropical regions.

\section{INTRODUCTION}

I T HAS LONG been suspected that a sufficiently strong initial disturbance is essential for creating a tropical storm of high intensity [5], [10]. In this letter, we have taken an approach to construct a hypothetical model of the creation of a strong initial disturbing vortex, which hitherto has not been considered in the literature. We have laid special emphasis on the large-scale uncertainty remaining involved in the whole process. This is by and large a very complex nonlinear phenomenon and, hence, possibly susceptible to chaotic behavior. But in this letter, we have applied techniques from fuzzy set theory (perhaps the first time in the atmospheric science) to present a simple model of the initial disturbing vortex. Due to the advantage inherent in the technique we can ignore the complexity due to nonlinearity to some extent and loose information accordingly. But this remarkably simplifies our essential task at hand of modeling the initial disturbing vortex out of a simple set of natural preconditions. In a finer and more sophisticated model, where information is progressively retained, this vortex will remain. Only more and more subtle attributes will have to be added to it.

In order to make a fuzzy model, we follow the classical convention of mathematical modeling of a physical phenomenon. Often proposing an appropriate set of differential equations for a climatic phenomenon is difficult. Even if such a set of equations is determined to some degree of accuracy, in many cases it may not be possible to supply exact values of parameters and variables as input to this system. In this case, fuzzy modeling is a very convenient tool. One very important thing to keep in mind here is that all kind of uncertainties cannot be dealt with using statistical models, because statistical models are based on classical two-valued logic. On the other hand, many-valued fuzzy logic is a much more versatile tool to deal with a broader class of uncertainties. Realization of this fact prompted Zadeh to create the fuzzy dynamical system as a tool to model very complex phenomena [2]. For a good exposition of fuzzy-set-theory-based techniques, fuzzy numbers, and fuzzy arithmetic, which have been frequently used in this letter, one may want to consult a standard text like [8].

Manuscript received August 7, 2002; revised January 28, 2003.

The author is with the Electronics and Communication Sciences Unit, Indian Statistical Institute, Calcutta 700108, India (e-mail: kaushik_r@isical.ac.in). Digital Object Identifier 10.1109/TGRS.2003.810674

\section{MODELING OF THE Disturbing VoRTEX}

Weather prediction experts are aware of the existence of several empirical conditions that are necessary but not sufficient for the formation of cyclones [5], [9]. The first of these requires the sea temperature to be at least $26^{\circ} \mathrm{C}$ through a depth of at least $60 \mathrm{~m}$. A second requirement is the absence of significant vector changes of the mean wind that extends into the troposphere. Relative humidity will have to be $85 \%$ or more for a long time throughout the region of storm formation and development. There are other empirical conditions as well, but even when they are satisfied, storm formation usually does not take place. In fact necessary climatic and geographical conditions for the formation of the tropical storms prevail over large areas of the earth during storm seasons, yet the actual occurrence of a storm is a relatively rare phenomenon. This indicates that there must be a rare coincidence of circumstances before development of a storm. The formation always occurs in connection with some kind of preexisting disturbance associated with a deep cloud layer. All of these disturbances do not intensify into tropical storms. Only a small percentage of these systems start intensifying. Numerous studies have been made to clarify the process of their formation, but no general mechanism has yet been accepted. In one such study described in [5], the evolution of the maximum surface wind speeds, with respect to time, in two of the experiments are compared. The first experiment starts with a maximum wind of $43.2 \mathrm{~km} / \mathrm{h}$. After about three days, the vortex rapidly intensifies to a nearly steady state amplitude of about $162 \mathrm{~km} / \mathrm{h}$. The second experiment is identical to the first, except that it was started with a maximum wind of only $7.2 \mathrm{~km} / \mathrm{h}$. The model storm in this case did not amplify even after $150 \mathrm{~h}$ had elapsed. Apparently, the model needs a sufficient "kick" (disturbance) to get a cyclone started [5], [10]. In nature, an asymmetric disturbance, such as an easterly wave, is almost always observed to precede the occurrence of tropical cyclones [5], [9].

To build the model of "a vortex initiated by a sudden disturbance," we take our clues from two-dimensional satellite images of matured cyclonic vortices. Though these features are of a matured cyclone, we accept them as clues toward the development of such shapes. It is natural to assume that the initial disturbing vortex is of log-spiral shaped strong wind vortex that is yet to draw large clouds from the surrounding areas to be prominent enough to be photographed from a satellite. We have taken the following features of such images to infer the initial stage.

- The clouds from the surrounding areas of the eye are curving inward to the eye in log-spiral shape. It is already recognized that the curved cloud bands surrounding the eye of a disturbance indeed take log-spiral shape [1], [4]. This is possible only if there was a preexisting wind vortex to draw clouds toward its center. 
- Here, clouds are taking shape along the drag of the wind converging to the eye. So, the wind tending to converge to the eye is supposed to traverse a log-spiral path in the vicinity of the eye.

In [5] and [10], to get a model cyclone started, a weak vortex, which decays upward from the surface, had to be superimposed on the basic state (of the model) of formation of the storm. The upward decaying effect may be ignored if the disturbance is considered to be limited within a certain layer of the atmosphere. We additionally assume that the wind drag toward the center of the vortex will be along log-spiral paths similar to that near the eye of a matured cyclone. Resolving the velocity of the disturbance or the "kick" (which is nothing but wind jet(s) or waves in reality) along the components of the cylindrical coordinates $(r, \theta, z)$ we get the following:

$\mathrm{dr} / \mathrm{dt}$ radial component;

$\mathrm{r}(\mathrm{d} \Theta / \mathrm{dt})$ cross-radial component;

$\mathrm{dz} / \mathrm{dt} \quad$ vertical component.

To get a log-spiral shape of the vortex, as seen from the top of the cylinder, i.e., along $z$ axis downward (negative $z$ direction), the following equation must be satisfied:

$$
\frac{\frac{\mathrm{dr}}{\mathrm{dt}}}{\mathrm{r}\left(\frac{\mathrm{d} \theta}{\mathrm{dt}}\right)}=\mathrm{m} \text { or } \mathrm{r}^{-1}\left(\frac{\mathrm{dr}}{\mathrm{d} \theta}\right)=\mathrm{m}
$$

where $\mathrm{m}$ is a constant.

We may assume that the disturbances propagate parallel to the ground, i.e.,

$$
\frac{\mathrm{dz}}{\mathrm{dt}}=0
$$

It is important to note that (2.1) and (2.2) give a simple dynamical model of only a disturbance leading to the development of a tropical cyclone. Equations (2.1) and (2.2) are valid only for a very short time at the very beginning of the genesis of a storm. Once the storm is formed, the model of the storm may be represented by a set of suitable equations like the ones in [3], [7], or [9].

Equation (2.1) can appropriately model only when $(\mathrm{r}, \theta)$ denotes the precise location of a point. But (2.1) is intended to model a vortex created by a certain wind jet or wave, where $(\mathrm{r}, \theta)$ denotes the position of an average point on the tip of the wind jet or wave creating the vortex. In reality, the location of such an average point cannot be determined with great precision. A substantial uncertainty will always remain involved that cannot be ignored.

Clearly, our model-based study of a climatic disturbance leading to the genesis of a storm is a knowledge-based system made up of vague or imprecise information. How does the vagueness or imprecision or uncertainty arise in (2.1)?

Well, here the value of $r$ depends on the value of $\theta$ ( $\theta$ is crisp, in fuzzy set terminology crisp means nonfuzzy) and the initial value $r(0)$. In practice, 1$)$ the value of $m$ will fluctuate even in short time intervals and 2) we cannot determine the initial value $\mathrm{r}(0)$ precisely. Uncertainties 1) and 2) propagate to the value of $\mathrm{r}$ calculated by solving (2.1). So, the uncertainty in determining the value of $r$ is the compound uncertainty due to 1 ) and 2).

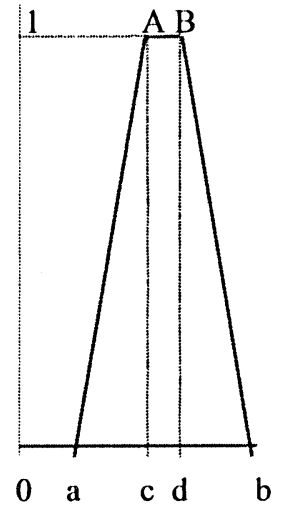

Fig. 1. Graph of the fuzzy constant $\mathrm{M}$ (the trapezium $\mathrm{aABb}$ ), which is a fixed trapezoidal fuzzy number. $\mathrm{m} \in[\mathrm{a}, \mathrm{b}]$. $\mathrm{m}$ takes values in $[\mathrm{c}, \mathrm{d}]$ with probability 1 .

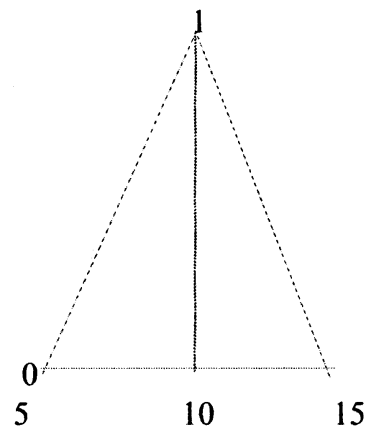

Fig. 2. Graph of fixed triangular fuzzy number $r_{0}$. Support of $r_{0}$ is $[5,15]$ in $50-\mathrm{km}$ scale, which is compatible with [9], $\mathrm{r}_{0}(10)=1$.

To accommodate these uncertainties we formulate (2.1) as a fuzzy differential inclusion (FDI) relation as follows:

$$
\mathrm{r}^{\prime}(\theta) \in[\mathrm{m} * \mathrm{r}(\theta)]^{\alpha}, \quad \mathrm{r}(0) \in\left[\mathrm{r}_{0}\right]^{\alpha} .
$$

The asterisk $(*)$ is the multiplication sign. If $\mu$ is a fuzzy set $\mu^{\alpha}$ denotes the $\alpha$ cut or the $\alpha$-level subset of the fuzzy set $\mu$. All fuzzy sets used in this letter are fuzzy numbers. That is, if $\mu$ is a fuzzy set, then $\mu$ is a mapping $\mu: \mathrm{R} \rightarrow[0,1]$, such that, there exists a closed bounded interval $[\mathrm{a}, \mathrm{b}]$ of $\mathrm{R}$ for $\mathrm{x} \notin$ $[\mathrm{a}, \mathrm{b}], \mu(\mathrm{x})=0 . \mu$ satisfies some additional conditions also [8], which are not important in this letter. Though in standard fuzzy set terminology $\mu$ is called a fuzzy set, actually $\mu$ is a function, which is like a probability distribution function used in measuring the fuzzy membership value. $0 \leq \alpha \leq 1$. By an $\alpha$-level subset of $\mu$, denoted by $\mu^{\alpha}$, we mean the subset of [a, b] (which will turn out to be a closed, bounded subinterval) given by $\{\mathrm{x} \mid \mu(\mathrm{x}) \geq \alpha\} . \mathrm{m} * \mathrm{r}(\theta)$, being an uncertain quantity, has been treated as a fuzzy number. The meaning of $[\mathrm{m} * \mathrm{r}(\theta)]^{\alpha}$ will become clear in the following paragraphs.

Next, we are to specify $\mathrm{m}$ and $\mathrm{r}(0)$. For any given value of $\theta$, $r(\theta)$ takes values in a fuzzy number. Fuzziness occurs because we cannot determine $\mathrm{r}(\theta)$ precisely for any given $\theta$. We are to take $r_{0}$ such that all possible crisp values of $r(0)$ always remain within it. We take $r_{0}$ as a triangular fuzzy number (Fig. 2).

We assume that for the sustenance of a stable storm m must take values from a closed, bounded interval $[a, b]$, whose interior is nonempty. $\mathrm{M}$ is such a fuzzy number that only consists of all feasible values of $\mathrm{m}$. By feasible values of $\mathrm{m}$, we mean only those values for which a stable vortex is possible to generate, 
whose intensity is "enough" to give rise to a tropical storm (may or may not be of hurricane intensity). We take $\mathrm{M}$ as a trapezoidal fuzzy number (Fig. 1).

$\mathrm{M}([-\infty, \mathrm{a}])=\mathrm{M}([\mathrm{b}, \infty])=\{0\}$, which means for values of $\mathrm{m}$ in $[-\infty, \mathrm{a}] \cup[\mathrm{b}, \infty]$ the generated vortices will not be strong and stable enough to give rise to a tropical storm of a given intensity, say T3, or more in Dvorak scale [4]. For values of $\mathrm{m}$ in $[\mathrm{a}, \mathrm{c}) \cup(\mathrm{d}, \mathrm{b}]$ (a, c, d, and b are to be determined experimentally) the vortices generated may still mature into a tropical storm but with a much lesser possibility. The greater is the membership value the more is the possibility of the generated vortex to mature into a tropical storm. When $\mathrm{m} \in[\mathrm{c}, \mathrm{d}]$ the possibility of cyclogenesis is the highest. Under favorable conditions (some of which we have mentioned in the first paragraph of this section) for $\mathrm{m} \in[\mathrm{c}, \mathrm{d}]$ a storm of hurricane intensity is very likely to be generated. A relatively small length of $[\mathrm{c}, \mathrm{d}]$ indicates the lesser possibility of generation of storms of very high intensity. This is of course a very simplified structure for M. Some other structure(s) for the fuzzy number $\mathrm{M}$ is (are) also quite possible.

\section{Simulation AND DiscuSsiON}

We have assumed that the variation of the initial disturbing wave along the $z$ axis is negligible and that it is only propagating parallel to the ground. So, FDI relation (2.3) is sufficient to represent the model. Let us restate (2.3) as

$$
\mathrm{r}^{\prime}(\theta) \in[\mathrm{m} * \mathrm{r}(\theta)]^{a}, \mathrm{r}(0) \in\left[\mathrm{r}_{0}\right]^{\alpha}, \mathrm{m} \in[\mathrm{M}]^{\alpha}
$$

where the fixed trapezoidal fuzzy number $\mathrm{M}$ is given by Fig. 1, and the triangular fuzzy number $r_{0}$ is given by Fig. 2 .

The solution of the ordinary crisp differential equation $\mathrm{r}^{\prime}=$ $\mathrm{m} * \mathrm{r}$ is dependent on $\mathrm{m}$ and $\mathrm{r}(0)$. Here, we shall consider the set of all such crisp solutions (union of whose graphs is termed integral funnel [6]), where $\mathrm{m} \in \mathrm{M}$ and $\mathrm{r}(0) \in \mathrm{r}_{0}$. A membership value will be assigned to each such crisp solution, where the membership value will be determined by the membership values of $m$ and $r(0)$. This way we get the fuzzy set of solutions of the differential equation $\mathrm{r}^{\prime}=\mathrm{m} * \mathrm{r}$, where $\mathrm{m}$ and $\mathrm{r}(0)$ are fuzzy valued. As a solution of (3.1) for a given $\alpha$, we take only those solutions $\mathrm{r}$ whose membership value $\geq \alpha$. Let us mention that (3.1) should have been written as $\mathrm{r}^{\prime}(\theta) \in\left[\mathrm{M} * \mathrm{r}_{0}\right]^{\alpha}$, which is the mathematically correct form. But this would have been more cumbersome to follow. The product of two fuzzy numbers is another fuzzy number [8].

Now we are in a position to simulate (3.1) according to the algorithm stated below. The simulated diagram represents the initial disturbing vortex. The size of this vortex will have great effect on the final intensity of the storm [3], [9].

1) replace $\in$ by $=$ in (3.1);

2) calculate the right-hand side of the equation thus obtained by Zadeh's extension principle [8];

3) fix $\alpha \in[0,1]$;

4) take the $\alpha$-level set on the right-hand side, which is an $n$-tuple of $\alpha$-cuts of fuzzy numbers for some $n$;

5) solve (directly or numerically) the ordinary crisp DEs only for the boundary values of the $\alpha$-level set;

6) the space enclosed by these solutions is the $\alpha$-level set of the solution of the equation obtained by replacing $\in$ by $=$
TABLE I

NuMERICAL SPECIFICATION OF THE FUZZY CONSTANT M

\begin{tabular}{c|c|c|c}
\hline $\mathrm{a}$ & $\mathrm{c}$ & $\mathrm{d}$ & $\mathrm{b}$ \\
\hline-0.2 & -0.1 & -0.05 & -0.001 \\
\hline
\end{tabular}

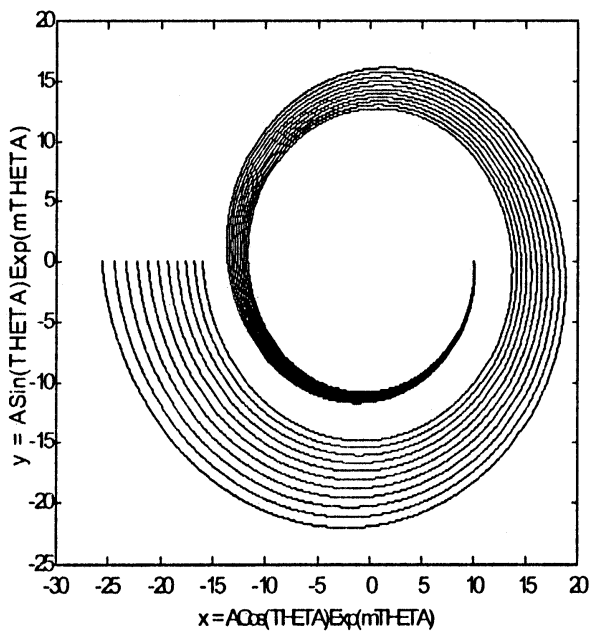

Fig. 3. Numerical simulation of (3.1) in 50-km scale, where $\alpha=1$, that is, the phase space is showing the fuzzy flow representing the best system behavior only, which resembles the generated initial cyclonic vortex. In this simulation of (3.1) $\theta$ has been taken as varying from $-3 \pi$ to 0 and the integration constant $\mathrm{A}=\mathrm{r}(0)$

in (3.1) and, hence, the solution of the FDI (3.1) for the given $\alpha$.

We have already mentioned in the previous section that the values of a, c, d, and b are to be determined experimentally. Here, we have determined them by computer simulations using synthetic data. It is rather an attempt to make a meaningful inference about the values of $a, c, d$, and b, which have been presented in Table I.

One very important thing to note here is that, $\mathrm{m}$ is taking negative values only. Mathematically, m could take positive values as well. But in that case, the orientation of the vortex would have been just the opposite. The vortex would not then be cyclonic but anticyclonic in northern hemisphere (in the southern hemisphere, the orientation will just be the opposite). Under the favorable influence of the coriolis force due to the rotation of the earth about its own axis the cyclonic vortices gather extra angular momentum to intensify. Therefore, in this letter we are concerned about the cyclonic vortices (of the northern hemisphere) only.

Now we are in a position to solve (3.1), where $\mathrm{M}$ is given by Fig. 1, and Table I and $\mathrm{r}_{0}$ is given by Fig. 2. For a direct solution of (3.1), we have to evaluate the product of two fuzzy numbers $\mathrm{M} * \mathrm{r}_{0}$ by Zadeh's extension principle [8]. Let $\mathrm{M} * \mathrm{r}_{0}=\mathrm{S}$, then

$$
\mathrm{S}(\mathrm{s})=\operatorname{supinf}_{\mathrm{s}=\mathrm{m} * \mathrm{r}(0)}\left(\mathrm{M}(\mathrm{m}), \mathrm{r}_{0}(\mathrm{r}(0))\right) \text {. }
$$

For any given $\alpha$, let $\mathrm{U}=\{\mathrm{s} \mid \mathrm{S}(\mathrm{s}) \geq \alpha\}$. Solve the crisp differential equation $\mathrm{r}^{\prime}=\mathrm{m} * \mathrm{r}$ for such $\mathrm{m}$ and $\mathrm{r}(0)$ that, $\mathrm{m} * \mathrm{r}(0)=\mathrm{s} \in \mathrm{U}$. This particular solution is an $\alpha$-solution, i.e., a solution with membership value $\alpha$ or more, of (3.1). Collection of all such $\alpha$-solutions of (3.1) will give the $\alpha$-flow of (3.1) (in Fig. 3 the 
1-flow is shown). This $\alpha$-flow is actually the fuzzy solution of (3.1), which signifies the behavior of the system given by (3.1) with possibility $\alpha$. The best system behavior is obviously given by the $\alpha$-flow, when $\alpha=1$. Fig. 3 gives the phase space of the best system behavior of (3.1). The flow shown in Fig. 3 tends to converge to a (fuzzy) point of the (fuzzy) phase space, which is known as the fuzzy attractor (fuzzy limit point to be more precise) of the system. If this vortex ultimately matures into a severe cyclonic storm this fuzzy attractor will be the eye of the cyclone.

The physical significance of the log-spiral shaped fuzzy flow of Fig. 3 is that, it represents the initial disturbance generating the initial vortex (like the ones in [5, Fig. 1]), which if sufficiently strong, under favorable conditions may develop into a cyclone. We have seen that the (fuzzy) log-spiral shape of the vortex is due to the (fuzzy) constant ratio between the radial and cross-radial components of the velocity of the disturbance. For a more straightforward interpretation for the generation of the initial vortex we may consider not one but two simultaneous disturbances, both of them are linear wind jets or waves coming from different directions and colliding and merging to produce the initial vortex. In this case, one of them acts as the radial component of the amplitude of the vortex and the other acts as the cross-radial component. Our mathematical model and the subsequent numerical simulations give a very realistic picture of this two disturbance hypothesis. For example, we have seen in [5] how a $43-\mathrm{km} / \mathrm{h}$ initial kick has ultimately generated a $162-\mathrm{km} / \mathrm{h}$ storm. If in our model we take this $43-\mathrm{km} / \mathrm{h}$ kick as the cross-radial velocity (acts in the anticlockwise direction in the northern hemisphere and hence positive) then we can calculate the radial velocity from $\mathrm{m}$. If we take $\mathrm{m} \in[-0.1,-0.05]$ so that $\mathrm{M}(\mathrm{m})=1$, the radial velocity must be in $[-4.3,-2.15]$ (in the southern hemisphere cross-radial velocity will be negative and $\mathrm{m}$ (and $\theta$ ) will be positive, i.e., the radial velocity will always be negative, which means that the wind and along with that also the clouds will always tend to converge toward the center of the vortex), which at any rate is a minor, almost negligible disturbance (the rate of convergence of cloud toward the cyclone will be slow, not more than about $5 \mathrm{~km} / \mathrm{h}$, initially). Note that the cross-radial velocity must be obtained from the major kick, i.e., the major kick must act tangentially to the vortex. The minor kick must act along the radius-vector of the vortex. This shows that our choice of $\mathrm{M}$ is very much realistic.

This model can also give satisfactory explanation of the phenomenon of occurring of the most intense cyclonic storms often either during April-May (just before the onset of the monsoon in the Indian subcontinent) or during October-November (just after withdrawal of the monsoon from the Indian subcontinent). During these times of the year major changes take place in the wind pattern over the seas surrounding the Indian coasts (Indian Ocean, Bay of Bengal, and Arabian Sea). The wind changes course from land-to-sea to sea-to-land (April-May) and from sea-to-land to land-to-sea (October-November). Naturally during these periods the possibility of simultaneous occurrence of one major and one minor disturbances as described above is very high and, hence, the relative high frequency of intense cyclones.
We have been able to establish through our model that climatic disturbances in the form of simple linear wind jets or waves are capable of creating log-spiral like cyclonic vortices. The role of such disturbances behind creation of cyclones have long been suspected [5]. This model is compatible with the model presented in [9]. Once the sufficiently strong initial cyclonic vortex is created according to our model, the development of the vortex toward maturity (i.e., a stable matured intense cyclone) can be simulated as given in [9]. Structure of such a storm in matured stage is given in [9, eq. (11)]. The manifold of this structure has been elaborated in [9, Fig. 1] in terms of constant angular momentum surfaces in the $\mathrm{r}-\mathrm{z}$ plane (r radius, $\mathrm{z}$ height), the real life significance of which has been illustrated in [9, Fig. 5]. [9, Figs. 1 and 5] pertain to the vertical cross section of the vortex of the storm. Fig. 3 of this letter, on the other hand, gives the horizontal cross section of the vortex of the storm, at the starting, near the surface of the sea. This model however is not compatible with [10], for we have not taken the velocity of the propagation of the disturbance during formation of a weak initial disturbing vortex according to [10, eq. (37)]. We have shown instead that the initial disturbing vortex can be generated in a more natural and simple way. Though we have proposed the model based on observations made in [5], [9], and [10], it is yet to be tested in real situation. The model can be improved by incorporating the thermodynamic and fluid dynamic considerations in the fuzzy constant $\mathrm{M}$ and considering the other solutions of (3.1) also along with the best possible ones (i.e., for which $\alpha<1$ ). This is one remarkable advantage of this type of fuzzy differential inclusion relation modeling. We can improve it just by accommodating more and more complex considerations in one single uncertain quantity M. M seems to have absorbed all lack of information to facilitate the vital task at hand to obtain the initial log-spiral shaped disturbing vortex out of strong linear wind jets or waves.

The development of the storm in the neutral case is an example of the finite-amplitude air-sea interaction instability described in [9]. In [9], it is argued that when entrainment and other buoyancy reducing effects are taken into account, the mean tropical atmosphere is very close to neutral, rather than conditionally unstable. Because of this, anomalous surface winds are necessary to enhance the surface heat fluxes. This energy is then redistributed in the vertical by convection, which then intensifies the large-scale circulation and the surface heat fluxes, and so on. When viewed in this context, the initial vortex must have a large enough amplitude to enhance the surface fluxes enough for the instability to occur [3]. Keeping this in mind we have taken $\mathrm{r}(0)$ about $500 \mathrm{~km}$, which is compatible with [9] and [10].

According to our model, the knowledge of the speed of wind, above certain threshold levels and coming from different directions over a region, can be utilized to detect the formation of a cyclone at a very early stage, even earlier than what we usually get today.

\section{CONCLUSION}

A modeling of a climatic phenomenon often involves a lot of uncertainties. One of the most uncertain areas in the modeling of a cyclone is its initial stage [3], [5], [7], [10]. It is well 
known that a sufficiently strong initial disturbance needs to be present to start a cyclone, even in the most favorable climatic and geographical conditions (some of these climatic conditions are described in the first paragraph of Section II, the geographical regions and storm seasons are well known), for formation of a storm [5], [10]. The immediate question is "How to model this initial disturbance?" We have tried to answer this question in this letter. To answer this question we have taken an hitherto unapproached path (Ooyama's model [7] was used previously to study the transformation of a wave into a closed vortex [11]), namely to make a log-spiral shaped cyclonic vortex out of strong linear wind jets or waves. This is compatible with observation, because tropical cyclones result from preexisting disturbances such as easterly waves [10]. Of course an initial cyclonic vortex may form in many other ways (like the ones mentioned in [7], [10], and [11]). The author believes that the way the formation of the initial cyclonic vortex has been modeled in this letter is one of the most natural possibilities of occurrence of such phenomena.

Recently, the author proposed a model of turbulence as chaotic occurrence of vortices in a dynamic fluid [12]. It has long been suspected that atmospheric turbulence has some role in the process of generation of storms. The model of generation of a storm as proposed in this letter and the model of turbulence as proposed in [12] have some common aspects leading to a support to the hypothesis that, atmospheric turbulence has some definite role in the generation of storms. The author has plans to further explore this area in short future.

\section{ACKNOWLEDGMENT}

The author's foremost acknowledgment goes to a little girl who was a 1999 Orissa supercyclone victim, whose plight drove him into this research. The author would like to thank two anonymous reviewers for their constructive criticisms and encouraging comments. The author is also thankful to D. P. Mukherjee for meticulously reading, criticizing, and editing the final manuscript.

\section{REFERENCES}

[1] P. Bouthemy and A. Benveniste, "Modeling of atmospheric disturbances in meteorological pictures," IEEE Trans. Pattern Anal. Machine Intell., vol. 6, pp. 587-600, 1984.

[2] M. De Glas, "Theory of fuzzy systems," Fuzzy Sets Syst., vol. 10, pp. 65-77, 1983.

[3] M. DeMaria and J. D. Pickle, "A simplified system of equations for simulation of tropical cyclones," J. Atmos. Sci., vol. 45, pp. 1542-1554, 1988.

[4] V. F. Dvorak, "Tropical cyclone intensity analysis using satellite data," NOAA Satellite Applications Lab., NOAA Tech. Report NESDIS 11, 1984.

[5] K. A. Emanuel, "Toward a general theory of hurricanes," Amer. Sci., vol. 76, pp. 370-379, 1988

[6] E. Hullermeier, "Numerical methods for fuzzy initial value problems," Int. J. Uncertainty, Fuzziness, Knowledge-Based Syst., vol. 7, pp. 1-23, 1999.

[7] K. Ooyama, "Numerical simulation of life-cycle of tropical cyclones," J. Atmos. Sci., vol. 26, pp. 3-40, 1969.

[8] G. J. Klir and B. Yuan, Fuzzy Sets and Fuzzy Logic: Theory and Applications. New Delhi, India: Prentice-Hall of India, 1997.

[9] K. A. Emanuel, "An air-sea interaction theory for tropical cyclones. Part I: Steady state maintenance,” J. Atmos. Sci, vol. 43, pp. 585-604, 1986.

[10] R. Rotuno and K. A. Emanuel, "An air-sea interaction theory for tropical cyclones. Part II: Evolutionary study using a nonhydrostatic axisymmetric numerical model," J. Atmos. Sci., vol. 44, pp. 542-561.

[11] V. Bliss, "Numerical simulation of tropical cyclone genesis," Ph.D dissertation, Univ. of Washington, Seattle, WA, 1980

[12] K. K. Majumdar, "Fuzzy fractals and fuzzy turbulence," IEEE Trans. Syst., Man, Cybernet. B, to be published. 